\title{
¿Por qué Paquito no sabe sumar?... cuarenta años después: ya suma pero no multiplica y menos divide
}

\author{
Why Paquito does not know how to add? ... forty years later: He already adds but does not multiply \\ and less divide
}

\section{Francisco Javier García ${ }^{a}$, María del Socorro Arredondo ${ }^{b}$, Alfonso Ávila Aguirre ${ }^{c}$}

\begin{abstract}
:
Numerical manipulation is essential to the daily existence that is one reason why the work focuses attention on the errors associated with the basic operations of high school students, is shocking the generality of the existence of such mistakes in a population that has spent at least six years in contact with the topic. To outline the possible reasons for this, a brief and historical description of textbooks, the training programs of the undergraduate students in Primary Education, the situations of the educational administration associated with the conditions for school failure and inadequate responses from high school students, of course others remain outstanding: teachers' beliefs, attitudes of students and family members towards school math, and more.
\end{abstract}

Keywords:

Educational Administration, Mistakes, Secondary Education

\section{Resumen:}

La manipulación numérica es esencial para la existencia diaria, esa es una razón del por qué el trabajo centra la atención en los errores asociados con las operaciones básicas de los estudiantes de la escuela secundaria. Es impactante la generalidad de la existencia de tales errores en una población que ha pasado por lo menos seis años en contacto con el tópico. Para bosquejar las posibles razones de ello, se hace una descripción breve e histórica de los libros de texto, los programas de formación de los estudiantes de la Licenciatura en Educación Primaria, las situaciones de la administración educativa asociadas con las condiciones para la reprobación y las respuestas inadecuadas de los estudiantes de primero de secundaria. Por supuesto, quedan pendientes otras: las creencias de los profesores, las actitudes de los alumnos y los familiares hacia la matemática escolar y más.

Palabras Clave:

Administración educativa, Errores, Educación Secundaria

Artículo recibido: 24 de julio de 2019

Dictaminado: 20 de septiembre de 2019

Segunda versión: 23 de octubre de 2019

Aceptado: 23 de octubre de 2019

\footnotetext{
a Autor de Correspondencia, Profesor-investigador de la Escuela Normal Superior del Estado de México, Email: pacoensem@gmail.com

${ }^{\text {b }}$ Profesora-investigadora de la Escuela Normal Superior del Estado de México, Email: macocoaz@gmail.com
} 


\section{Introducción}

En el perfil de egreso de la primaria se declara que el alumno: "Comprenda conceptos y procedimientos para resolver problemas matemáticos diversos..." (Gob.mx $\mathrm{s} / \mathrm{f})$ para lo que es indispensable "aprender a leer, escribir y hacer cuentas". A mediados de la década de los setentas (hace 40 años), Kline (1976) señalaba el por qué Juanito no sabía sumar, sin embargo Kline se refería a las respuestas consideradas erróneas por los maestros, porque a los estudiantes se les pedía justificar formalmente hechos aritméticos en términos de la matemática moderna, pero las

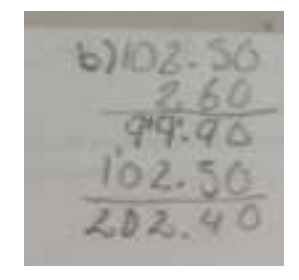

Figura 1. respuestas obtenidas de los algoritmos de la suma eran adecuadas. El problema actual es que los alumnos de la escuela secundaria (quienes han pasado por lo menos siete años en los salones) tienen confusiones con la multiplicación, véase la figura 1. Es la respuesta de un alumno de primero de secundaria, se trata de multiplicar 102.50 por 260. En su respuesta, para obtener 99.90, suponemos, hizo lo siguiente: $0-0=0 ; 5-6=9$ y “llevamos 1", $(1+2)=3$, $2-3=9$ y "llevamos 1"; $10-1=9$, a continuación, sumó 99.90 con 102.50 , cuyo resultado es 202.40 .

Desafortunadamente, la magnitud del problema es más grande de lo imaginado. Es común que los estudiantes de primero de secundaria tengan confusiones con las operaciones básicas, de ello, los maestros de las escuelas secundarias de Toluca, en los Consejos Técnicos Escolares, acordaron dedicar los primeros minutos de sus clases de matemáticas a la revisión de las operaciones.

Suponemos que las causas de las respuestas inadecuadas de los estudiantes son conjunciones de diversos factores, así, revisamos los libros de texto, los programas de formación de los estudiantes de la Licenciatura en Educación Primaria, las situaciones político-administrativas de las condiciones del trabajo de los maestros, y mostramos ejemplos de las respuestas impropias de los estudiantes de primero de secundaria. En los noventa se reportó que los niños a quienes la vida de todos los días les imponía hacer operaciones numéricas tenían éxito empleando sus propios algoritmos (Carraher, Carraher y Shliemann, 1991), el inconveniente aparecía en los salones de clase; el señalamiento de Carraher y Shliemann, que nos parece importante, está en afirmar que la escuela formal no había sido capaz de guiar la enseñanza tomando como base las formas naturales de los estudiantes para operar con los números. En su reporte, los niños resuelven adecuadamente las operaciones con sus algoritmos personales, pero no lo hacen con las reglas escolarizadas, donde implica "llevar", además la colocación inadecuada vinculada con el orden de las magnitudes numéricas son causa de respuestas equivocadas.

Por supuesto que hay discusiones acerca de la conveniencia de enseñar y aprender procedimientos para desarrollar cada una de las cuatro operaciones de la matemática escolar (suma, resta, multiplicación y división). En contra de tales procedimientos, se dice que son carentes de significado, sólo son automatismos, involucran únicamente a la memoria y fáciles de olvidar y confundir. A favor se afirma: son producto de la cultura histórica, son aplicables con independencia de los números particulares y son procesos eficientes (Sánchez, 2014, 11-13).

Con relación a la matemática escolar, los equívocos vinculados a las operaciones, la tendencia se inclina hacia la propia educación matemática (Anghileri, 1989; Hiebert 1986). También existen propuestas sobre la enseñanza de la aritmética escolar, resultado de la investigación en educación (Godino, 2004; Castro, Rico y Castro, 1988). Los estudios que han incluido los juegos en ambientes de computadora, comparados con la enseñanza con lápiz y papel, sugieren que no hay diferencias significativas en los logros académicos (Sayan, 2015).

Con referencia a otros aspectos asociados con la matemática escolar, tenemos los reportes de las 
investigaciones internacionales cuyas conclusiones señalan situaciones desventuradas: Los alumnos no asimilan los conocimientos, ni desarrollan las competencias en la asignatura. Reconocen signos y estructuras, pero con escasa capacidad para resolver problemas matemáticos simples de la vida cotidiana. (Casassus y otros, 2000: 13). En este estudio, se incluyeron otras variables, tales como: apoyo de la familia, habilidad propia de los alumnos, autoestima, ambiente de la escuela, recursos de la escuela, métodos de enseñanza y expectativas de los docentes. De ellas, las opiniones del maestro que supone que los resultados académicos dependen de las características cognitivas de sus alumnos y que sus propios métodos de enseñanza están asociadas con éxitos académicos; en cambio, los maestros que piensan que los logros académicos son responsabilidad de las familias, sus alumnos tienen resultados escolares inadecuados (Casassus y otros, 2000: 20).

Respecto a la trascendencia de las operaciones, en la vida de todos los días, conviene pensarlas como "ideas fundamentales", como procedimiento y como herramienta asociada con las situaciones problema que coadyuva a resolver situaciones reales. Así, la matemática escolar se concibe como una terna formada por: objetos de la matemática escolar (los números), las operaciones (manipulación) y las relaciones (mayor o menor qué) aplicables en la vida diaria de los sujetos. La matemática escolar de la educación básica, pensada como "matemática formal", la entendemos como un conjunto de objetos para los cuales se definen operaciones y se establecen relaciones. Por ejemplo: Los números reales (que es el conjunto que contiene a todos los demás conjuntos numéricos que se estudian en la educación básica), tenemos dos operaciones la suma y el producto, así como la relación de orden; en las geometrías damos existencia a los puntos, las rectas y los planos, y la relación es la de "pertenencia" y las operaciones son las transformaciones. En otras palabras, una operación binaria es una asociación de una pareja de números con un tercer número: $A$ la pareja $(2,3)$ le asociamos el 5 bajo la suma; y al 6 bajo la multiplicación. En la matemática escolar, a diferencia del lenguaje natural, nos interesa hacer afirmaciones y que éstas sean verdaderas, pero la verdad no es del tipo factual, la verdad que nos interesa es del tipo lógico. La conclusión (verdadera) es el resultado de una serie de afirmaciones (también verdaderas).

La forma de asociación entre una pareja de números y el tercero le llamamos algoritmo, procedimiento, también se entiende como regla de asociación. El problema señalado por Carraher es la diferencia entre los procedimientos escolares y los construidos por los estudiantes; el ejemplo de la vida de todos los días consiste en pagar con un billete que supera el costo de alguna mercancía y "el cambio, el vuelto" se calcula completando la cantidad, no usamos el algoritmo de la resta. Supongamos que pagamos $\$ 55.00$ con un billete de $\$ 100.00$, el encargado completa físicamente con monedas o billetes: " $55,56,57,58,59,60,70,80,90$, 100". Tal algoritmo se usa por los niños en las escuelas mexicanas desde los primeros grados, "tengo $n$ ¿cuánto me falta para $m$ ?' y los chicos usan los dedos para "completar". La situación descrita muestra la clase de problemas que se pueden resolver pensando: "tengo $n$ ¿cuánto me falta para $m$ ?", $n+x=m, x$ representa "lo que falta", en cambio, en la escuela la transformamos en: $x=m-n$, que ya no representa una acción física "natural".

\section{Método}

La información se adquirió de los estudiantes del séptimo y octavo semestres de la Licenciatura en Educación Secundaria con Especialidad en Matemáticas (nueve) de la Escuela Normal Superior del Estado de México (Ensem). La educación de la escuela Normal se conoce como "formación inicial", el propósito es que se apropien del trabajo de los maestros en sus múltiples facetas, las materias de las licenciaturas se agrupan en tres áreas de actividad (Plan 1999), estos son: Las escolarizadas: acercamiento a la práctica escolar y práctica en condiciones reales de trabajo.

En los dos últimos semestres, esas tres áreas se unen en actividades fundamentales, y son: 
- El desarrollo del trabajo docente con varios grupos de alumnos de educación secundaria.

- "El diseño y administración de propuestas didácticas en los grupos escolares; el análisis y la reflexión sistemática acerca de su desempeño con los grupos de alumnos que atienden, así como, sobre el conjunto de experiencias obtenidas en la escuela secundaria" (SEP, 2002, 7).

- La elaboración del documento recepcional (similar a una tesis).

Los registros escritos son de los escolares de la escuela secundaria y que son alumnos de los estudiantes de la Normal. Las diferentes escuelas secundarias están en la ciudad de Toluca, o cercanas, las comunidades donde se ubican son: San Pablo Autopan, La Maquinita, El Seminario y Metepec. Los registros se tomaron de los cuadernos de los estudiantes de la secundaria, las preguntas y problemas que generaron los escritos son parte de las clases de todos los días. Hay otra información que fue obtenida de las grabaciones en audio de los alumnos de la Normal durante las discusiones acerca de sus experiencias en las escuelas de práctica. El trabajo es de tipo descriptivo y se trata de comprender las formas de pensar de los sujetos, determinadas por diferentes grupos de factores.

Suponemos que las respuestas inadecuadas a las tareas escolares son producto de esquemas mentales (en términos de Piaget), impropios. Así, el análisis de tales respuestas son indicadores de las falsas concepciones y confusiones de los sujetos. Conviene conocer la lógica asociada a los errores o a sus fuentes porque puede guiar acciones encaminadas a la disminución o supresión de ellos.

\section{Discusión}

Libros de texto. Con relación a las posibles fuentes de los errores por supuesto están los libros de texto (LT). Enseguida hacemos una breve revisión histórica de éstos, para la enseñanza y el aprendizaje de la matemática escolar.

Las ideas subyacentes de los métodos de enseñanza han pasado por supuestos sobre el aprendizaje como los textos tipo catecismo, donde la enseñanza se hacía a través de preguntas y respuestas (Beyer, 2019). Otra

forma de enseñanza fueron los casos particulares generalizados. Por ejemplo: Cuando en la multiplicación de dos números, uno de ellos está formado por dígitos consecutivos se hace la suma conservando las magnitudes de los dígitos, 57 multiplicado por 23 se suma dos veces el 57 en "las decenas" y tres veces "en

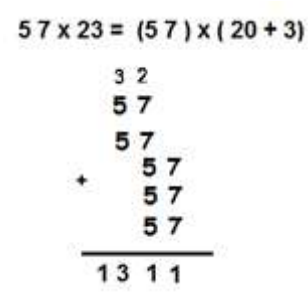

Figura 2.

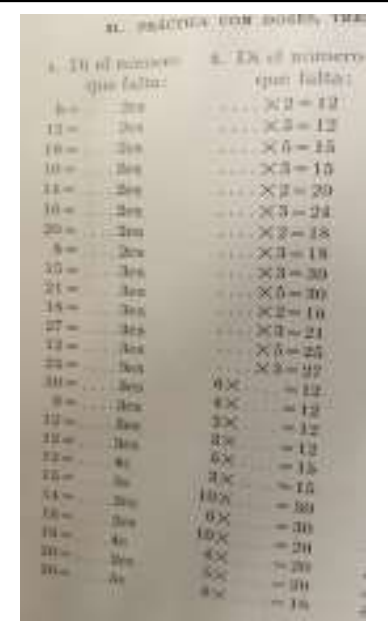

Figura 3.

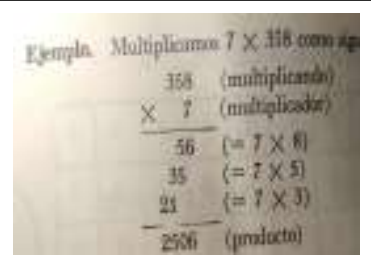

Figura 4.

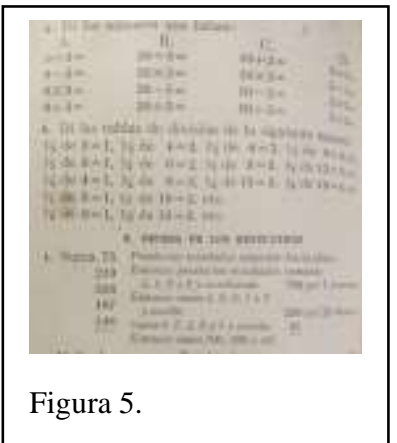

las unidades". La operación se ilustra en la figura 2. Otro ejemplo "para multiplicar un número por seis, se divide al número por dos, se multiplica por diez y se suma el número original". La explicación del porqué funciona esta regla es la expresión: $\quad 6 \times n=$ $\frac{n}{2} \times 10+n=5 n+n$.

También se ha empleado la repetición junto con la memorización de los pasos para el desarrollo de los algoritmos (Thorndike, 1924; ver figura 3). Además se le ha dado énfasis a los significados de las operaciones, como en la matemática moderna (Kline, 1976). Pero también, los algoritmos "típicos" se han usado "siempre". La figura 4 corresponde al libro de Zubieta y Sánchez (1960) En los libros citados y empleados en los salones de clase, los temas son desarrollados en orden de dificultad. Thorndike (1924, 1) inicia pidiendo al estudiante contar verbalmente "hasta donde 
puedas", continúa con la escritura de los números "uno, dos,... hasta donde puedas", la sección termina con la escritura simbólica de los números "hasta donde puedas". Las operaciones con números se inician con una cifra, se siguen con dos cifras, etcétera.

En las lecciones combina las operaciones y pide verificar los resultados, figura 5 . La intención de Thorndike (1926) es que el estudiante identifique patrones de comportamiento de los diferentes grupos de ejercicios.

Los libros de texto editados por la SEP (2019), tienen una propuesta que nos parece extraña (figura 6). La página corresponde al libro de segundo año de primaria, es la segunda actividad del bloque I, está en la página 13. En seguida resolvemos el problema.

\section{La rifa}

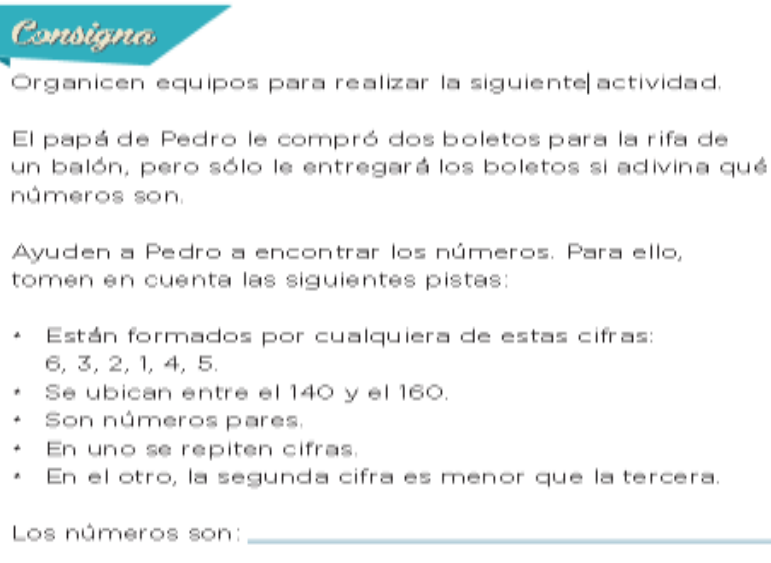

Figura 6

1. Los números son de tres cifras, la cifra de las centenas es 1 (porque "se ubican entre el 140 y el 160").

2. La cifra de las unidades es 2, 4 o 6 (porque "son números pares", es la primera vez que en estos grados, primero y segundo de primaria, se mencionan a los números pares, y se da por hecho que los estudiantes ya saben que son aquellos números "que terminan" en 2, 4, 6, 8 ó 0 o que son de la forma $2 n$.

3. Así que tenemos números de tres cifras, donde la primera cifra de las centenas es 1 , y la cifra de las decenas es mayor o igual a 4, eso excluye al 2 y 3 porque tendríamos ciento veinte... y ciento treinta... que son menores a 140 , excluimos al 6 porque el número de los boletos no es mayor a 160 .
4. La lista de números que tenemos con las condiciones anteriores son: 142; 144; 146; 152; 154; 156

5. Uno de los números es 144 ("en uno se repiten las cifras")

6. Hay dos números que cumplen con la condición "la segunda cifra es menor que la tercera", 146 y 15 6.

Consideramos que el problema anterior no es adecuado para los estudiantes de segundo año de primaria, básicamente por la falta de experiencia matemática de los niños. Además, en el mismo libro, en la página 88, se pregunta: “¿Cuántos números diferentes de dos cifras se pueden formar con las siguientes tarjetas?" En la ilustración están los números 5,7 y 4, (son 9 números), es un problema de conteo, que es un prerrequisito al problema de la figura 6 de la página 13 . Suponemos que la propuesta de enseñanza de los libros de texto actuales corresponde a la intención de que los estudiantes den sentido a los problemas. Sin embargo, no está bien lograda, además, la secuencia para iniciar la construcción del significado de número real a través de los diferentes números: Naturales, Fracciones, Decimales, Racionales y otros como $\pi$ (Freudenthal, 1983, 133-177), en los niños no parece tener alguna estructura clara.

La magnitud de los números es otro problema conceptual olvidado en los libros de texto de la SEP. El sentido numérico, cuyo desarrollo se observa en la solución de situaciones problema, requiere de la experiencia de diversas tareas, como contar uno a uno, además llevar un registro numérico con los dedos. Otros factores asociados con los números son: la experiencia con diversas representaciones y criterios acerca del sentido relativo y absoluto de las magnitudes numéricas (como la afirmación 1 anterior), y estar consciente de la posibilidad de la existencia de más de una solución (Zuhal 2017; Tracey 2012), es obvio de ello, que el éxito de los alumnos en la tarea de la figura 6 sea nula.

Compárense las imágenes de las lecciones de los libros de la SEP (2019), la primera es el libro para el alumno de 1972. La segunda, la edición para el maestro de 2013. Esperar, en 2013, que con esa información los alumnos “... relacionen la posición de las cifras con sus 
valores...", es una apuesta que seguro se pierde. Los alumnos requieren mucha más experiencia con situaciones que involucran acciones que les permita interiorizarlas y generar representaciones y no sólo la información, eso lo saben "los maestros de banquillo".

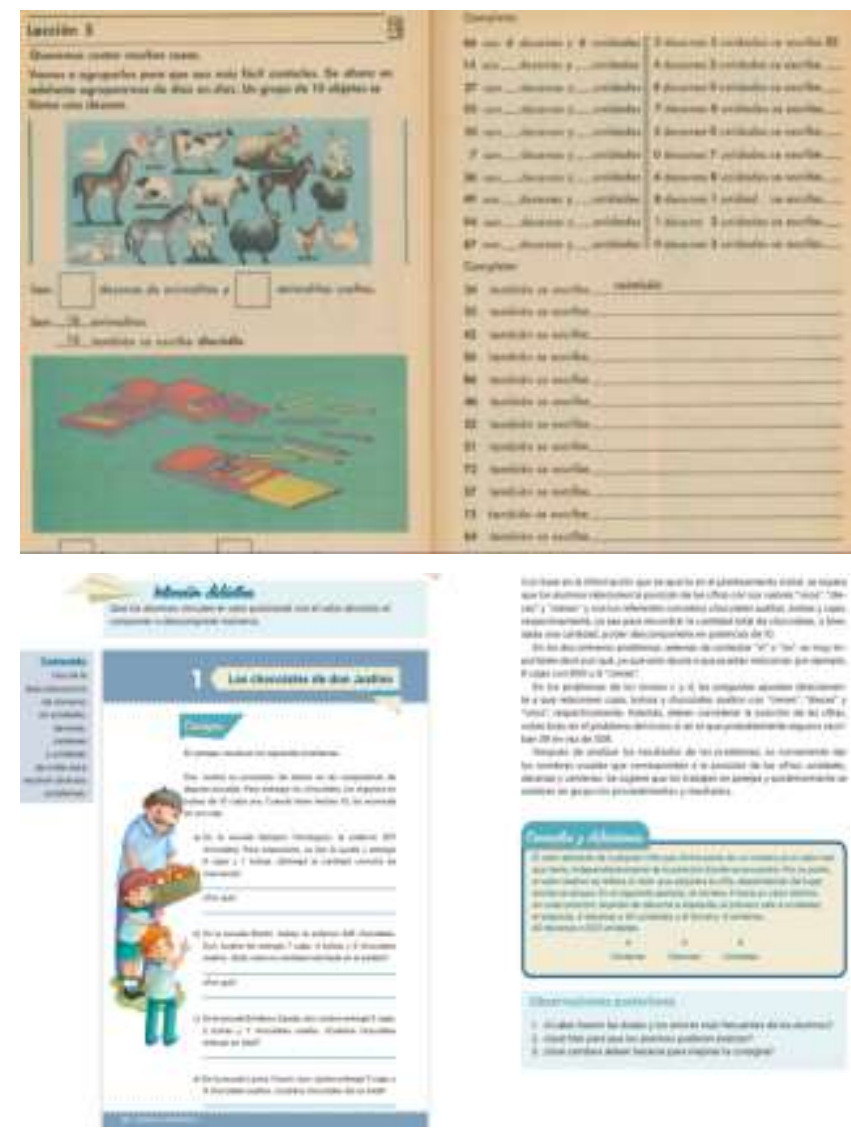

Los programas de estudio de la licenciatura en educación primaria. Plan 2012 (SEP 2013, 16).

En los programas de estudio de la Licenciatura en Educación Primaria. Plan 2012 (SEP 2013), la bibliografía está centrada en las formas de enseñanza y el aprendizaje de los números, sólo hay una referencia en la bibliografía complementaria sobre las matemáticas como especialidad (College Algebra. Rider P. Original de 1940). La razón por la que creemos necesario enfatizar la matemática, es por el hecho que los estudiantes conocen la matemática de la escuela preparatoria y ésta enfatiza los procesos algorítmicos, y la matemática "interesante" es diferente. Una forma de describirla es como un tipo particular de Lenguaje.

Una parte fundamental de cualquier lenguaje es el significado asociado a las proposiciones (afirmaciones verdaderas o falsas), y las proposiciones tienen significado específico en un lenguaje determinado, fuera de ese lenguaje, el significado puede ser completamente diferente o puede no existir. Un lenguaje formal como el de la matemática tiene las siguientes características:

- Objetos (como los números o los puntos, rectas y planos).

- Reglas para formar nuevos objetos, de tal forma que los objetos sean objetos bien escritos.

- Proposiciones iniciales que se aceptan como verdaderas (axiomas).

- Reglas que permiten transferir la verdad de los axiomas a nuevas proposiciones (reglas de inferencia)

- Definiciones, nombres para proposiciones complejas (afín, límite).

- Teoremas (son afirmaciones derivadas de las afirmaciones iniciales).

El lenguaje de la matemática escolar es un lenguaje formal, aunque los puntos anteriores no se hagan explícitos a los maestros en su formación inicial. Los sistemas de representación de la matemática escolar (SRME) tienen elementos cardinales de construcción: reglas de formación, reglas de inferencia, definiciones y sintaxis. Una idea que subyace en los SRME es la asociación entre el objeto de la matemática escolar, y su representación, dicho de otra forma, hay una relación directa entre el significado y el significante (Fregoso, 2007, 15-38).

No proponemos resucitar a la matemática moderna en la formación inicial de los maestros, pero conviene mediar la construcción de los objetos importantes, como el de los diferentes números y los diferentes algoritmos, a fin de cuentas se trata de propiciar la concepción de los objetos sus operaciones y sus relaciones de matemática entre los maestros.

\section{La administración educativa y las condiciones reales de la escuela.}

Otro origen de los resultados no deseables con la matemática escolar son las condiciones y decisiones político-administrativas de los administradores de la educación básica. Por cada estudiante reprobado o en riesgo de reprobación, es decir, con calificaciones de seis, al maestro le exigen un proyecto de intervención 
para ser desarrollado en los periodos de recesos de clases (con anterioridad eran vacaciones de verano), en los meses de julio y agosto, esas fechas están señaladas en el calendario escolar. Los maestros siguen considerando esos periodos "sus vacaciones", además el proyecto de intervención por cada alumno genera trabajo complicado que necesita tiempo y recursos propios de los docentes.

En los casos de los alumnos reprobados y en riesgo de reprobación, el maestro está obligado a escribir un reporte con argumentos y "evidencia" que justifique las calificaciones reprobatorias. Para la elaboración de tales reportes no hay periodos determinados, cada director decide cuándo y en qué tiempo el maestro tendrá que entregarlo, no es raro que se le pida de un día para el siguiente.

Debido a la exigencia de atender a los estudiantes en riesgo, las respuestas que han dado los profesores son: Trabajar con los alumnos reprobados o en riesgo de reprobación durante los periodos de recreo entre las clases. Sin embargo, Nay (alumna de la Normal) expresa: "desafortunadamente los que asisten son los alumnos que no tienen problemas". Hay otros maestros que deciden asignar temas a los estudiantes, con la intención de que los expongan ante todo el grupo. En ocasiones, en tales exposiciones, se les pide a los padres participar en ellas; otra de las estrategias para prever los resultados es construir guías para el examen, pese a ello, el número de respuestas correctas en los exámenes es lamentable. Esto en palabra de Gp, se expresa de la siguiente manera.

Gp: Hubo niños que en el examen tuvieron un acierto de cuarenta y cinco. Esos ejercicios (los del examen) fueron los mismos que hicimos en clase; en clase trabajan bien, y participaban, tenían dudas y preguntaban, y en el examen salieron mal, muy mal,... el maestro (responsable del grupo) les dijo que corrigieran su examen tres veces (escribieran tres veces las respuestas correctas), para subir la calificación porque no había otra manera de hacerlo. Lo que hice con los padres fue sacar su calificación (en la reunión donde fueron convocados) con la escala (firmas, sellos, trabajos extra). Tenían calificaciones de 3 , $4.2,0.1$. Los papás preguntaban (incrédulos), si esa era su calificación, las firmas eran 130 y sólo tomamos 100, tampoco pasaban; los papás les decían "¿a qué vienes a la escuela?" y otros, nada mencionaban, y me preguntaban: '¿qué puede hacer?' Les decía que tenían que traer su corrección y uno la traía... Como no los podemos reprobar, los vamos a poner a hacer algo; como no tenían firmas (tareas revisadas), los niños medio las entregaban, y no era la única materia. Sí me sentí frustrada, había pasado una semana y no entregaban, ya habíamos pasado calificaciones. El maestro les puso 7 a todos, le protesté porque no traían nada, dijo que no iba a trabajar con personas que no les interesaba estudiar. Como tres niños de cada salón no entregaron nada, aunque sus papás hayan ido a la reunión. No sé qué pase con los alumnos, pero no muestran interés o responsabilidad, no sé qué sea.

\section{Los errores escritos de los alumnos de la escuela} secundaria. Los errores asociados con las confusiones de cómo hacer los algoritmos.

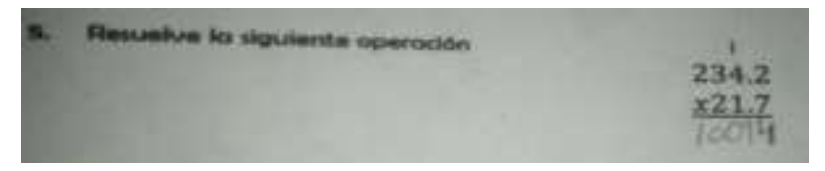

Figura 7.

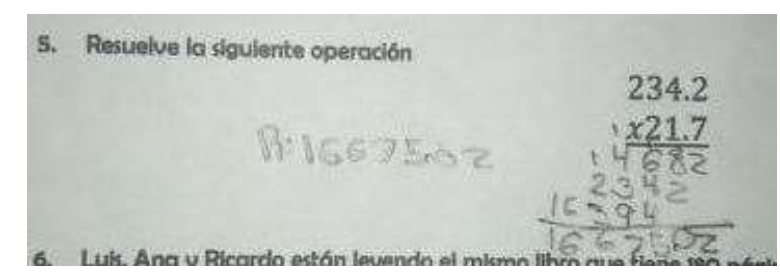

Figura 8.

En la figura 7, el estudiante operó los números y parece que mezcló las operaciones. La figura 8 sugiere que el niño tiene idea de cómo colocar los productos parciales, pero las multiplicaciones "individuales" le generan problemas. Sabe qué tiene "que llevar", sin embargo, no lo hace adecuadamente, como tampoco los productos. 
La última suma, la de los tres productos parciales, es correcta.

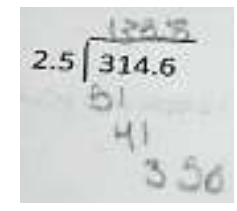

Figura 9.
En la figura 9, la primera cifra del resultado es correcta, pero no cada residuo parcial, falta información acerca de cómo piensa la división: Cómo reparto o cómo hago grupos de grupos, o una resta reiterada.

Sin embargo, no parece tomar consciencia que cada resultado parcial es mayor que el dividendo. Con sólo los registros es muy complicado averiguar cómo obtuvo cada resultado parcial.

Li reporta lo sucedido con un alumno en su clase, "me acerqué y le pregunté si sabía dividir, el niño dijo: sí. Le dije, pues haz la división. Pasó un rato y no hacía nada, le aseguré que no sabía dividir, dijo que no sabía y le dediqué un rato a explicarle".

Otro problema está asociado con los números decimales (figura 10), además de que las multiplicaciones individuales (excepto la multiplicación por 1) y los resultados parciales no son correctos. La colocación final de punto decimal genera problemas con la magnitud del resultado.

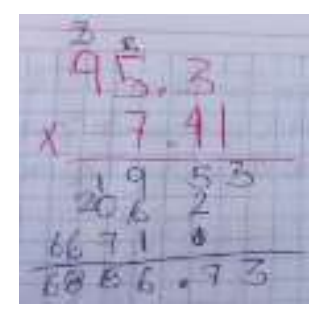

Figura 10.

Los registros son indicadores de que las concepciones cualitativas tanto de la multiplicación y la división no parecen existir. Es decir, la suma puede ser representante de la acción de agregar elementos individuales a una colección y la multiplicación como "agregar grupos" a una recopilación (figura 11).

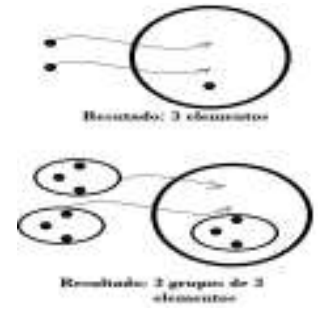

Figura 11.

En la división, como acciones físicas e interiorizadas como "repartir en grupos". Es decir, la enseñanza empleando representaciones de objetos manipulables es un posible camino para la formación del sentido numérico (Milton, y otros 2019).

Un posible camino para enfatizar las relaciones entre los números y dejando de lado por el momento el punto decimal. En la operación de figura 10, en una primera aproximación, podría considerarse como formar 7 grupos de 95 cada uno, eso generaría una magnitud como de 700, número lejano a los 6800 del resultado final. La división de la figura 9, con relación a los residuos parciales, se podría verificar en el sentido de comparar "51 es como 2 grupos de 25, así que aún cabe", como indicador de plausibilidad.

Una hipótesis de los registros de los estudiantes, es que las operaciones están pensadas y enseñadas como procedimientos, en los cuales no hay una distinción cualitativa entre ellos, sólo son formas de proceder. Por ello, son una colección de reglas aprendidas de memoria, donde comparten pasos como "llevar" pero los estudiantes no disponen de pistas para saber si cada paso es adecuado.

Otras respuestas impropias surgen de situaciones problema. Con relación a las operaciones, los estudiantes no tienen referencias de qué pueden significar los números; en los problemas verbales hay condiciones para establecer relaciones con los números.

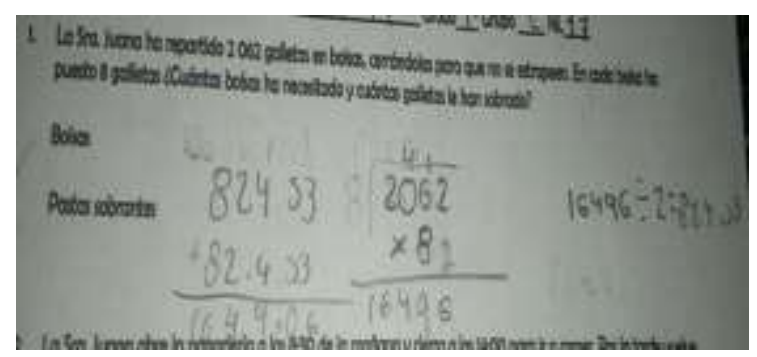

Figura 12. 
La figura 12, muestra que el estudiante sabe que requiere dividir 2062 entre 8, (el registro está parcialmente borrado), pero tiene problemas con la división, eso lo sabemos por el resultado de dividir 16496 entre 2 de la misma figura, obtiene 824.53, tanto 16486 como 2 son divisibles entre 2. Por ello, el resultado de la división es exacto. Es decir, el residuo de la división es cero y el resultado final no tiene decimales, caso contrario al registro.

Por otra parte, la inadecuada manipulación del punto decimal genera cambios de magnitud numérica e induce errores. Puede ser que para el alumno es suficiente que los números tengan los mismos dígitos con independencia de la magnitud que representan.

Por supuesto que si los estudiantes tienen ideas inadecuadas con las operaciones básicas en el ámbito de los "números que nos sirven para contar" (los Naturales), en el conjunto numérico de las fracciones (Racionales), las confusiones se incrementan exponencialmente, obsérvese la figura 13, la suma de las tres fracciones es 9 entre 9 (un entero) y éste es igual a 8 entre 7 que es mayor que un entero, es decir, un número es igual a otro que es más grande que él mismo.

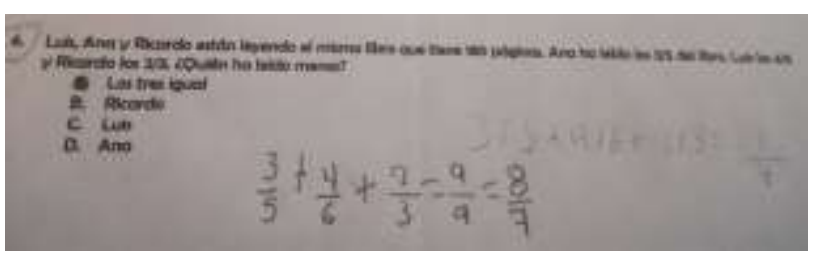

Figura 13.

Entre los problemas con los Racionales (quebrados) la definición de suma induce una clase de equivalencia, es una función, además, es un algoritmo y también la operación suma. En este conjunto numérico hay diversos significados de la expresión $\frac{a}{b}$, con $b \neq 0, a, b \in$ $N$ : es un número, es una división, es una definición, es operador, y una razón de cambio entre otros significados. Además, las acciones físicas, representadas por los números racionales, pierden significados, explicar y pretender que los niños de secundaria entiendan, con objetos físicos, que el resultado de la división: $\frac{7}{3} \div \frac{1}{4}=\frac{28}{3}=9 \frac{1}{3}$, sea mayor que el número "dividido" es inaceptable, en otras palabras, cuando tenemos un poco más que dos enteros (7/3) y los "partimos" en cuartos, el resultado es mayor a 9 "enteros", ese resultado está fuera de la intuición de los escolares.

\section{Conclusiones}

Hemos querido señalar que las respuestas de los estudiantes de la escuela secundaria tienen diferentes orígenes, cuyas inter-conexiones se ven reflejadas en las respuestas a los problemas y las operaciones: multiplicación y división.

Por una parte, los libros de texto de la escuela primaria hacen supuestos sobre las formas de aprendizaje y enseñanza, las cuales son poco adecuadas. Suponen conocimientos que no poseen los alumnos, porque no se les han enseñado, o porque hacen falta más experiencias de aprendizaje con esos tópicos. Carecen de "rutas" explícitas, comparadas con las de los libros de texto de los años setenta.

Convendría revisar los tópicos para la formación inicial de los maestros de la escuela primaria, en términos de contenidos de la propia matemática, la matemática desde la matemática, y por supuesto, hace falta definir qué contenidos y con qué formalidad (el rigor es incuestionable). Es obvia la existencia de elementos fundamentales e indispensables para la enseñanza de la matemática, como saber qué es un número Natural, un Racional o un Entero; qué es una operación y sus propiedades, saber qué es una función, qué es medir, entre muchos otros aspectos. Es recomendable que tales ideas formen parte del conocimiento general de todos los maestros, no para convertirlas en objetos de enseñanza, pero sí para considerarlas como guías para ella.

Bajo las condiciones político-administrativas actuales, de exigencia y control hacia los maestros, es materialmente imposible reprobar a los alumnos con independencia de sus logros académicos; afirmación que puede responder por los resultados de las evaluaciones externas, las cuales han sido poco exitosas. 
Los ejemplos presentados, sobre los registros de los alumnos, son muestras de la necesidad de promover la revisión de las formas, los medios y supuestos sobre la enseñanza y el aprendizaje de las operaciones matemáticas básicas, y de los criterios para centrar el énfasis en los logros académicos de los alumnos, más que en poner énfasis en las estadísticas administrativas. Finalmente, es importante señalar que conviene centrar la atención en la alfabetización numérica que necesita y que le es indispensable a todo egresado de educación básica, ya que se convierte en un elemento central para el desarrollo del pensamiento matemático. De la misma manera, esta premisa resulta aplicable a los maestros y estudiantes en las escuelas normales para maestros.

Si bien existen intentos por resolver los problemas que se presentan en los centros escolares, como el Sistema de alerta temprana (SisAT) y los Consejos técnicos escolares (CTE), ambos con materiales escritos y guías, estos no han tenido los resultados esperados. Creemos que existen conexiones e interdependencias entre diversos grupos de factores, cuyo abordaje "inteligente", tal vez disminuya los fracasos con las operaciones básicas y la solución de problemas textuales escolares elementales.

\section{Referencias bibliográficas y fuentes consultadas}

Anghileri, J. «An investigation of young children's undestanding of multiplication.» Education Studies in Mathematics, 1989: 367 - 385.

Beyer, Walter. «Scielo.» Catecismos y matemáticas: confluencia de corrientes de pensamiento. 26 de Enero de 2019. Disponible en: http://ve.scielo.org/scielo.php?script=sci_arttext\&pid=S101122512009000100007

Carraher, Terezinha, David Carraher, y Analucía Shliemann. En la vida diez, en la escuela cero. México. D.F.: Siglo XXI, 1991.

Casassus, J, S Cusato, J Froemel, y J. Palafox. Laboratorio Latinoamericano de evaluación de la calidad de la Educación. Primero estudio internacional comparativo sobre lenguaje, matemáticas y factores asociados para alumnos del terrecer y cuarto grado de la educación básica. Santiago de Chile: UNESCO, 2000.

Castro, E, J Rico, y E Castro. Números y operaciones. Madrid: Síntesis, 1988.

Fregoso, Arturo. Saber y Creer. Textos y pretextos sobre el pensamiento científico y el pensamiento religioso. Volumen III. . Texcoco: Universidad Autónoma de Chapingo, 2007.

Freudenthal, Hans. Didactical Phenomenology of Mathematical Structures. Dordrecht: D.Reidel Publishing Company, 1983.

Gob.mx. «Perfil de egreso de la educación obligatoria.» Perfil de egreso de la educación obligatoria. s/f de s/f de s/f. Disponible en: https://www.planyprogramasdestudio.sep.gob.mx/index-perfil-educobligatoria.html (último acceso: 26 de Septiembre de 2019).

Godino, Juan. Didáctica de las matemáticas para maestros. Enseñanza y aprendizaje de las matemáticas. Granada. España: Universidad de Granada, 2004.

Hiebert, J. Conceptual and procedural knowledge: The case of mathematics. Nuew Jersey: Lawrence Erlbaum Associates, 1986.

Kline, Morris. El fracaso de la matemática moderna. México: S XXI, 1976.

Milton, J, Margaret Flores, Alexcia Moore, Ja'Lia Taylor, y E Megan. «Using the concrete-Representational-Abstract Secuence to Teach Conceptual Understaning of Basic Multiplication an Division.» Learning Disability Quarterly. V42 n1, 2019: 32-45.

Sánchez, Framit, Manuel Rafael. «Univresidad de Granada.» Algoritmos en operaciones básicas: Alternativas, materiales y recursos en el aula de matemáticas. 30 de Junio de 2014. Disponible en: http://digibug.ugr.es/bitstream/handle/10481/36185/FramitSanchezRaf aelManuel.pdf?sequence=1 (último acceso: 2 de Mayo de 2019).

Sayan, Hamlyet. «The effects of computer games on the achievement of basic mathematical skills.» Educational Research anf Reviews. v10 n22, 2015: 2846-2853.

SEP. «Centro de descargas.» Desafíos Matemáticos Segundo grado 20172018. 13 de Mayo de 2019. Disponible en: https://www.cicloescolar.mx/2018/01/desafios-matematicos-segundogrado-2017.html.

SEP. «CONALITEG.» CONALITEG. 14 de Mayo de 2019. Disponible en: https://historico.conaliteg.gob.mx/content/common/consulta-libros$\mathrm{gb} /$ generacion.jsf ?busqueda $=$ true $\&$ nivelEscolar $=2 \&$ grado $=3 \&$ materia $=$ \&editorial $=\&$ tipo $=\&$ clave $=\&$ titulo=\&autor $=\&$ selectedyear $=1972 \&$ ani $\mathrm{o}=1972$.

SEP (2013) Licenciatura en educación primaria. Plan de estudio 2012.Segundo semestre. México: SEP, 2013.

SEP (2002) Lineamientos para la organización del trabajo académico durante el séptimo y octavo semestres. Licenciatura en educación secundaria. México, D.F.: Secretaría de Educación Pública, 2002.

SEP (2016) Orientaciones para la organización y usos del tiempo en la jornada escolar de una escuela de tiempo completo. México: SEP, 2016.

Thorndike, Eduardo. The Psycology of Aritmetic. New York: Macmillan, 1926.

Thorndike, Eduardo. Las aritméticas de Thorndike. Nueva York: Rand McNally, 1924.

Tracey, Muir. «What is Reasonable Answer? Ways for Students to Invetigate and Develop Their Number Sense.» Australian Primary Mathematics Classroom V17 N1, 2012: 21-28.

Zubieta, F, y E Sánchez. Aritmética razonada. Méxicio: IPN, 1960.

Zuhal, Yilmaz. «Young Children's Number Sense. Development: Related Complexity acrooss Cases of Tree Children.» Intenational Electronic Journal of Elemtary Education, v9 n4, 2017: 891-902. 